\section{Influence of different merging angles of pedestrian flows on evacuation time}

\author{
Manuela Marques Lalane Nappi, \\ Ivana Righetto Moser, \\ João Carlos Souza
}

\begin{abstract}
Architecture and Urbanism Program, Federal University of Santa Catarina, Florianópolis, SC, Brazil
\end{abstract}

\begin{abstract}
The growing number of fires and other types of catastrophes occurring at large events highlights the need to rethink safety concepts and also to include new ways to optimize buildings and venues where events are held. Although there have been some attempts to model and simulate the movement of pedestrian crowds, little knowledge has been gathered to better understand the impact of the built environment and its geometric characteristics on the crowd dynamics. This paper presents computer simulations about pedestrians' crowd dynamics that were conducted based on the Social Force Model. The influence of different configurations of pedestrian flows merging during emergency evacuations was investigated. In this study, 12 designs with different merging angles were examined, simulating the evacuation of 400 people in each scenario. The Planung Transport Verkehr (PTV, German for Planning Transport Traffic) Viswalk module of the PTV Vissim software (PTV Group, Karlsruhe, Germany) program was adopted, which allows the employment of the Social Force approach. The results demonstrate that both symmetric and asymmetric scenarios are sensitive to the angles of convergence between pedestrian flows.
\end{abstract}

\section{Introduction}

The growing numbers of fires and other types of catastrophes occurring at large events highlights the need to rethink safety concepts and also to include new ways to optimize buildings and venues where events are held. ${ }^{1}$ Moreover, the importance of the design of exit areas has been growing due to global tendency toward mass urbanization, mega events, terrorism and natural disasters. ${ }^{2}$ In this respect, studies on the pedestrian dynamics have been able to predict that small architectonic characteristics of the surroundings can have great effects on the behavior of crowds as well as on the pedestrian flow.
It is known that the higher the density, the more difficult it is to organize crowds, ${ }^{4}$ and thus the current field of pedestrian simulation has wide reaching areas of application. One of these relates to research on the movement of crowds using the Social Force Model, ${ }^{5}$ and the Models Based on Activities ${ }^{6}$ can be used to simulate all movement base cases and most self-organization phenomena. ${ }^{4}$ The Social Force Model is based on the modelling of fluid crowds. ${ }^{5,7-9}$ It is based on the superposition of attraction and repulsion effects, which are responsible for determining the behavior of individuals.

Although there have been some attempts to model and simulate the movement of pedestrian crowds, little knowledge has been gathered to better understand the impact of the built environment and its geometric characteristics on the crowd dynamics. ${ }^{10}$ On the other hand, it is known that the exact methods combined with the potential of computers are capable of producing results that are relevant to society. ${ }^{11}$ This aspect directly relates to the objective of this study, in which computer simulations of the crowd dynamics of pedestrians were conducted based on the Social Force Model and the influence of different configurations of the merging of pedestrian flows during emergency evacuations was investigated.

\section{Pedestrian flows and merging angles}

The planning and design of the interior of large installations, such as shopping centers and football stadiums, are very complex. Originating from this complexity is the equally complex behavior of crowds, where rotating movements, crossing and merging inevitably occur. Thus, it is important to understand the consequences of these collective movements before planning the spaces destined to host large numbers of people. ${ }^{12}$

The complex interaction between varied flows can lead to completely unexpected results, attributed to the nonlinearity of the pedestrian dynamics. This means that the planning of installations for pedestrians through conventional methods does not always manage to avoid heavy congestion and serious obstructions and blockages, especially when dealing with emergency situations. ${ }^{11}$ The Social Force Model can be used to reproduce self-organizing phenomena in pedestrian crowds, which have generally been neglected. ${ }^{9}$ The aim of this model is to develop design elements that increase the efficiency and safety of installations used by pedestrians.

The computer simulations, after calibration with empirical data on pedestrian flows, produce realistic results even when
Correspondence: Manuela Marques Lalane Nappi, Architecture and Urbanism Program, Federal University of Santa Catarina, 88040900, Florianópolis, SC, Brazil.

Tel. +55.48.991381473.

E-mail: 1alppi.ms@gmail.com

Acknowledgements: the authors would like to thank PTV Group for the academic license provided for the use of the PTV Viswalk software program.

Key words: Emergency evacuation; Fire; Computer simulation; Symmetric merging angles; Asymmetric merging angles.

Contributions: the authors contributed equally.

Conflict of interest: the authors declare no potential conflict of interest.

Funding: this study was carried out with financial support provided by the Coordination for the Improvement of Higher Education Personnel - Coordenação de Aperfeiçoamento de Pessoal de Nivel Superior (CAPES) Financial Code 001 - 88887.091740/2014-01 - PROALERTAS Project.

Conference presentation: part of this paper was presented at the $5^{\text {th }}$ Iberian-LatinAmerican Congress on Fire Safety (CILASCI $5^{\text {th }}$ Congress), 2019, July 15-17, Porto, Portugal.

Received for publication: 28 August 2019.

Accepted for publication: 23 September 2019.

This work is licensed under a Creative Commons Attribution 4.0 License (by-nc 4.0).

${ }^{\circ}$ Copyright: the Author(s), 2019

Licensee PAGEPress, Italy

Fire Research 2019; 3:75

doi:10.4081/fire.2019.75

considering new geometries and situations. Thus, the simulation software program adopted in this study, which is based on the Social Force Model, has predictive capability, allowing the investigation of new scenarios. It should be noted that, for several scenarios, experiments would involve high costs and be difficult or dangerous to carry out. The predictive capability is particularly important with regard to the planning and optimization of escape routes. ${ }^{9}$

In order to better understand the importance of the design and the merging angle in the behavior of large pedestrian crowds, previous studies have been conducted to investigate the influence of some merging configurations on the indexes for the response to escape routes under emergency situations. The results obtained confirm the role of the geometric characteris- 
tics of convergence layouts; however, further issues associated with this theme still need to be studied. It has been observed that both symmetric and asymmetric layouts are sensitive to the angles between the flows which are merging. However, the relation between the escape rates and merging angle is not monotonic, indicating the importance of the evaluation of convergence design and its influence on evacuations under emergency conditions. ${ }^{10}$

\section{Materials and Methods}

In this study, 12 designs with different merging angles were examined, simulating the evacuation of 400 people in each scenario analyzed. The PTV Viswalk module of the PTV Vissim software program was adopted, which allows the employment of the Social Force approach. In this approach, pedestrians can walk around regardless of their destinations, without a predefined network model for the direction in which they move. The software program, which adopts a microscopic model, allows the realistic simulation of pedestrians and models the human walking behavior, making it possi- ble to analyze pedestrian flows inside and outside buildings. ${ }^{13,14}$ Furthermore, it adopts as a standard the shortest path between the origin and destination of a pedestrian, which was maintained in this study.

It is important to note that the Social Force simulation model was selected based on the analysis of several studies ${ }^{15-18}$ as well as on the wide acceptance of this model in the academic arena. ${ }^{19}$ This model can be used for several collective behaviors, such as waves of stop-and-go, the faster is slower effect and arching and blocking. ${ }^{7,20}$

The scenarios simulated in this study were repeated for two different desired speeds (ds). The input speeds for each proposition were $1.32 \mathrm{~m} / \mathrm{s}$, which corresponds to a normal walking speed within a crowd, ${ }^{9}$ and $2.5 \mathrm{~m} / \mathrm{s}$, which corresponds to the walking speed of people in a hurry (faster than 1.5 $\mathrm{m} / \mathrm{s}$ ), introducing the parameter known as nervousness to the simulations. ${ }^{13}$

Considering these two speeds, the configurations totaled 24 scenarios, which were simulated 10 times each. Due to the stochastic nature of the simulation model adopted in this study, random fluctuations are expected, which can influence the results of the simulations carried out. The stochastic simulation models therefore have a random component which is not controlled by the researcher. ${ }^{21}$ Thus, for comparison purposes, the average of the maximum evacuation times for each scenario was calculated.

For the merging of pedestrian flows, five asymmetric configurations $\left(45^{\circ}, 60^{\circ}\right.$, $90^{\circ}, 120^{\circ}$ and $\left.135^{\circ}\right)$ and seven symmetric configurations $\left(45^{\circ}, 60^{\circ}, 90^{\circ}, 120^{\circ}\right.$ and $135^{\circ}$, $180^{\circ}$ and $270^{\circ}$ ) were analyzed, as shown in Figures 1 and 2, respectively.

It can be observed in Figures 1 and 2 that each configuration presents two branches that merge at the angles indicated. The points of origin of the pedestrians in the simulations are represented by green areas and the exit point is indicated in red. In the asymmetric configurations one branch is straight and the other is angular in relation to the direction of the exit. In the symmetric configurations, the two branches meet the direction of the exit at the same angle. Each point of origin releases, concomitantly, the same number of pedestrians into the network.
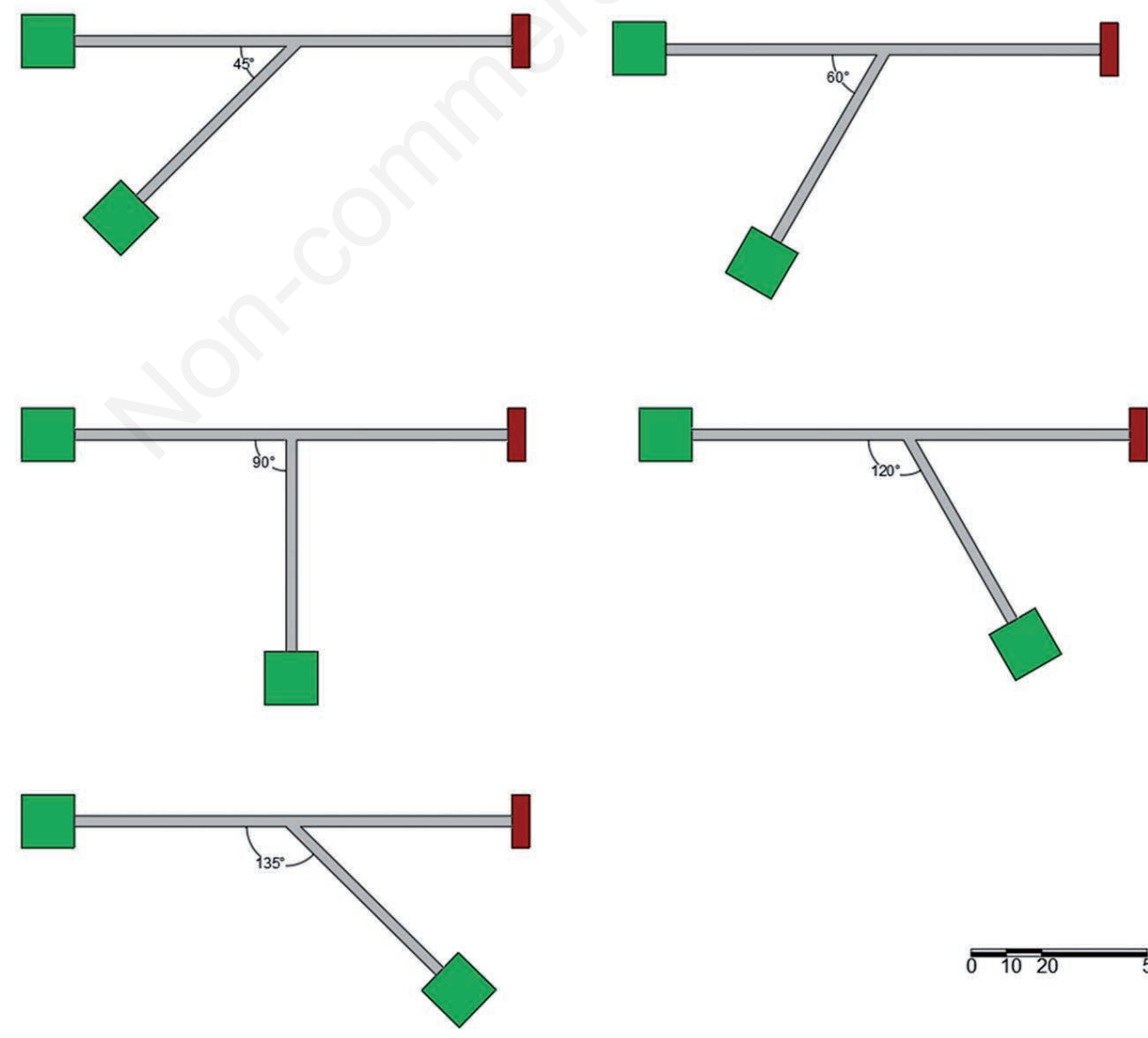

Figure 1. Asymmetric scenarios. 


\section{Results and Discussion}

In the asymmetric scenarios, the speed for the straight branch is faster in all situations analyzed, which results in a longer evacuation time for the angled branches (Figure 3). The difference between the evacuation times for the two branches becomes greater with an increase in the merging angle, for both speeds analyzed, as can be observed in Figures 4 and 5. In the symmetric scenarios, the pedestrian flows in the two branches which merge are relatively similar (Figure 6).

Considering the total evacuation time for each scenario, the correlations between the time and the merging angle were found to differ for the symmetric and asymmetric scenarios (Figures 7 and 8). In the asymmetric scenarios, the evacuation time tends to increase with an increase in the angle while in the symmetric scenarios the evacuation time oscillates with a slight tendency to decrease with an increase in the merging angle.

It can also be observed in Figures 7 and 8 that the asymmetric scenarios show a slightly better performance regarding the total evacuation time, considering a normal walking speed $(1.32 \mathrm{~m} / \mathrm{s})$. However, when the speed corresponding to nervousness $(2.5$
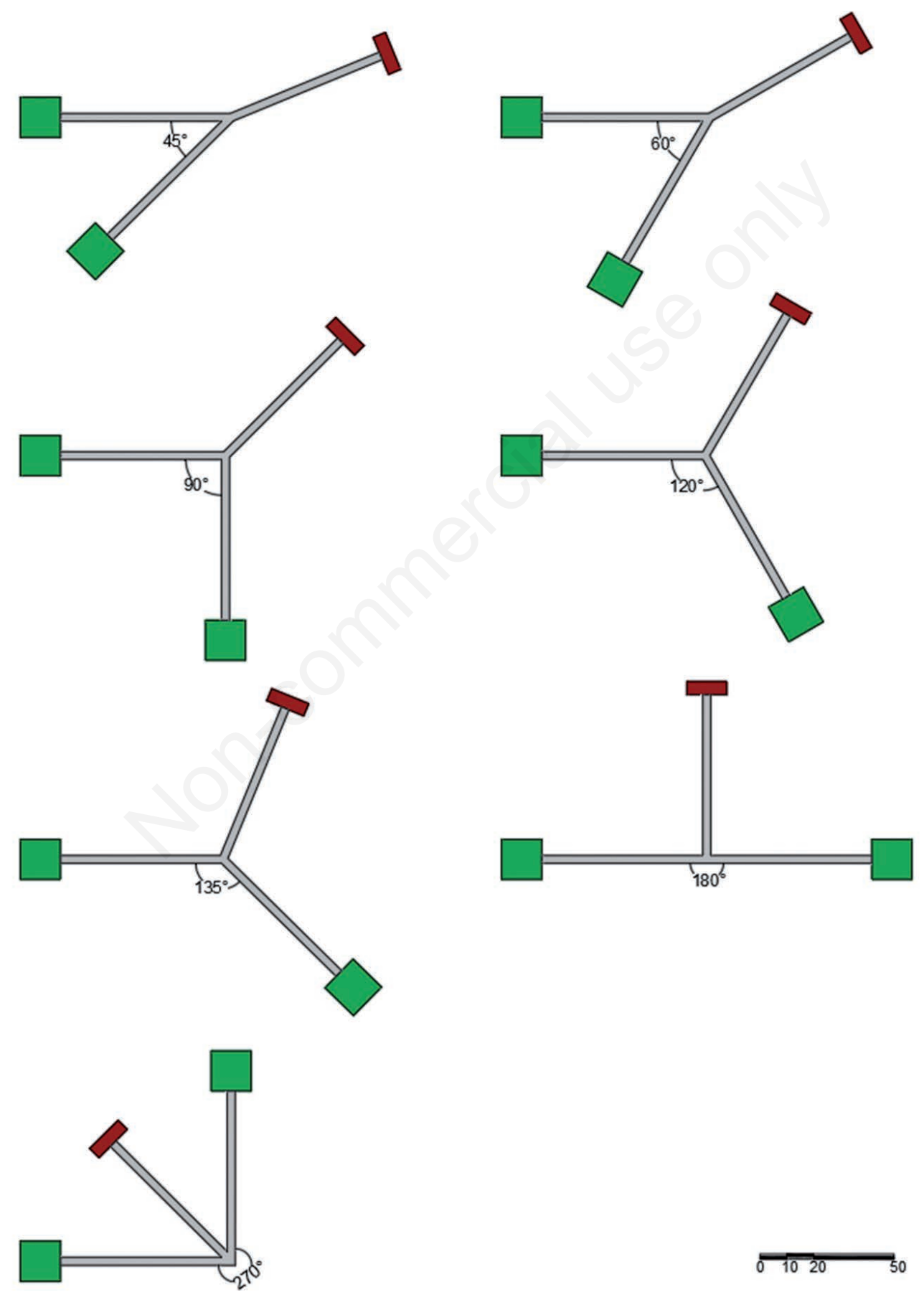

Figure 2. Symmetric scenarios. 
$\mathrm{m} / \mathrm{s}$ ) is analyzed, the obtuse merging angles $\left(120^{\circ}\right.$ and $\left.135^{\circ}\right)$ in the symmetric scenarios show better performance than the same angles in the asymmetric scenarios. In addition, a brief analysis of the results obtained for the acute angles and straight routes demonstrates that the difference between the evacuation times for the symmetric and asymmetric scenarios tends to decrease with an increase in the escape speed.

As can be seen in Table 1, for the normal walking speed $(1.32 \mathrm{~m} / \mathrm{s})$, the total evacuation times were slightly longer for the symmetric scenarios compared with the asymmetric scenarios. However, similar average evacuation times were observed when both types of scenarios were simulated at the speed of $2.50 \mathrm{~m} / \mathrm{s}$.

\section{Conclusions}

The results obtained demonstrate that both symmetric and asymmetric scenarios are sensitive to the angles of convergence between pedestrian flows. In the asymmetric scenarios, there is an increase in the evacuation time with an increase in the merging angle between a straight line and an angular branch. In this regard, the symmetric scenarios show oscillating results, however, with a slight tendency toward a reduction in the evacuation time with an increase in the merging angle. In general, the asymmetric scenarios present a slightly better performance regarding the total evacuation time, considering normal walking speed $(1.32 \mathrm{~m} / \mathrm{s})$. However, there are indications that with an increase in the evacuation speed, the symmetric merging is more effective. In conclusion, the analysis carried out verifies that the planning of the geometric characteristics of escape areas can play an important role in optimizing the flow during the evacuation of pedestrians.
Table 1. Average of the maximum evacuation times for each simulated scenario.

\begin{tabular}{lcccc} 
Merging angle & $\begin{array}{c}\text { Normal walking } \\
\text { Asymmetric }\end{array}$ & $\begin{array}{c}\text { Sls=1.32 m/s }) \\
\text { Symmetric }\end{array}$ & $\begin{array}{c}\text { Hurried walking } \\
\text { Asymmetric }\end{array}$ & $\begin{array}{c}\text { Symmetric } \\
\text { Sym }\end{array}$ \\
$45^{\circ}$ & 246.33 & 251.96 & 94.76 & 95.78 \\
$60^{\circ}$ & 245.43 & 253.27 & 94.72 & 95.65 \\
\hline $90^{\circ}$ & 246.06 & 252.04 & 95.04 & 95.22 \\
$120^{\circ}$ & 248.66 & 254.68 & 97.56 & 96.24 \\
$135^{\circ}$ & 250.49 & 251.60 & 98.43 & 95.45 \\
$180^{\circ}$ & - & 246.87 & - & 94.86 \\
\hline $270^{\circ}$ & - & 260.35 & - & 101.78 \\
Averages & 246.33 & 252.97 & 96.10 & 96.43 \\
\hline
\end{tabular}

ds, desired speeds.

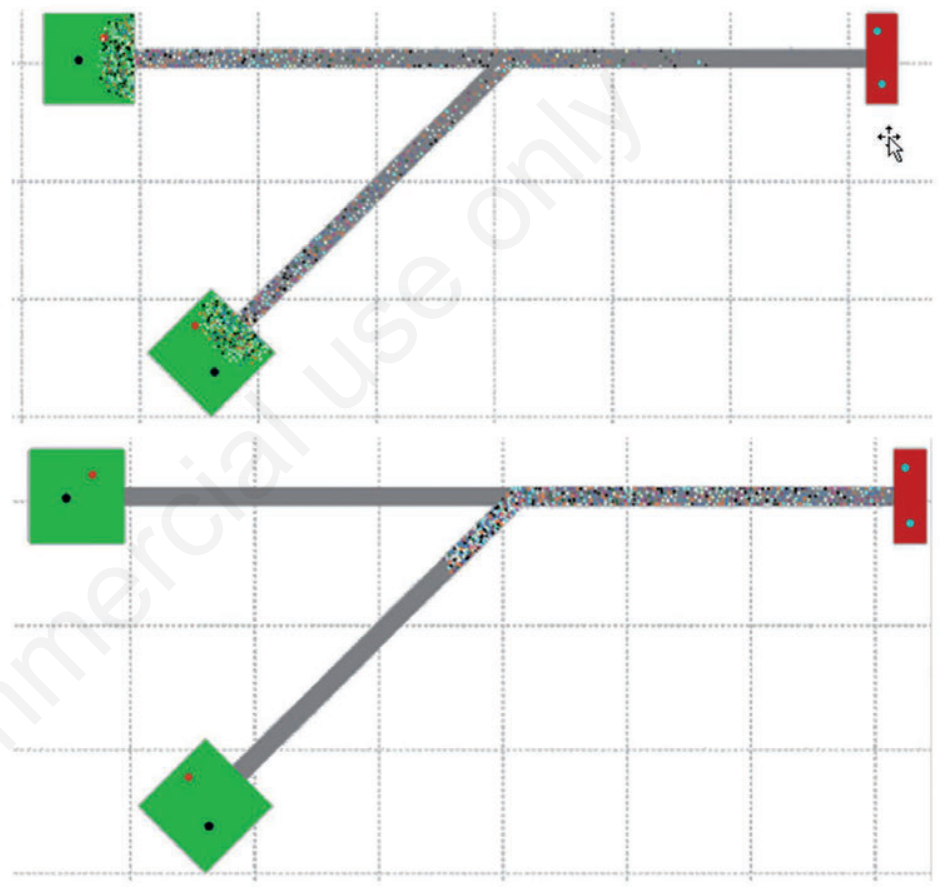

Figure 3. Screen grabs of an asymmetric scenario at $35 \mathrm{~s}$ (top) and $84 \mathrm{~s}$ (bottom) from the start of the simulation.

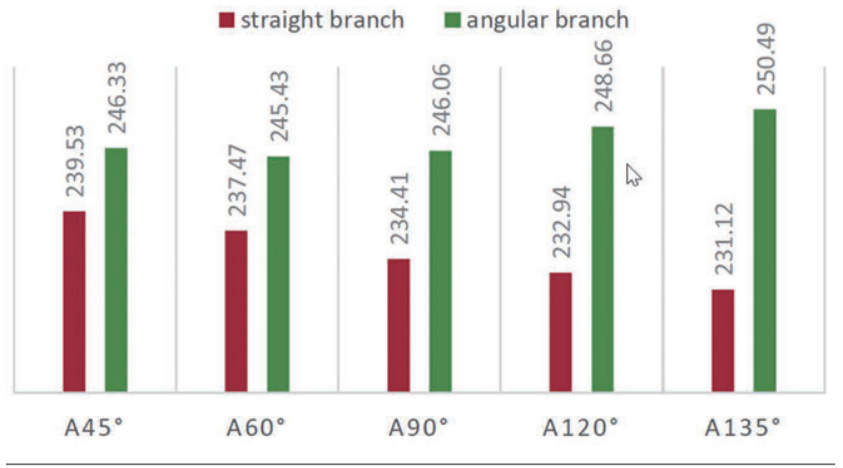

Figure 4. Evacuation time of the asymmetric scenarios for the speed $S 1$.

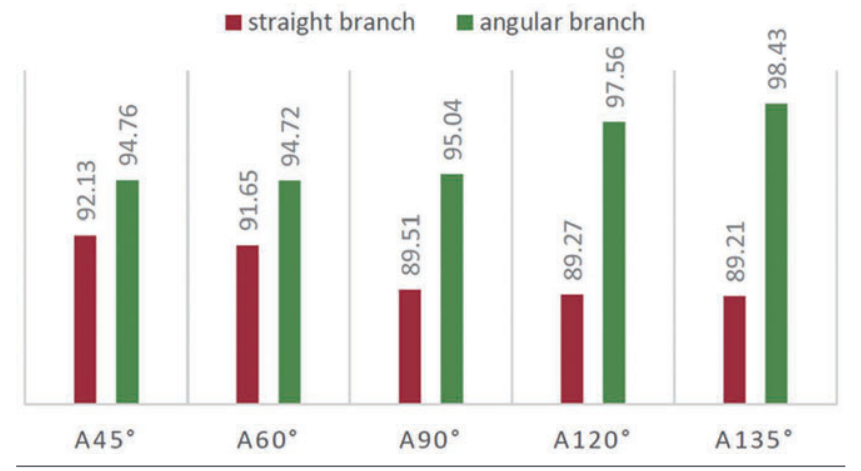

Figure 5. Evacuation time of the asymmetric scenarios for the speed S2. 


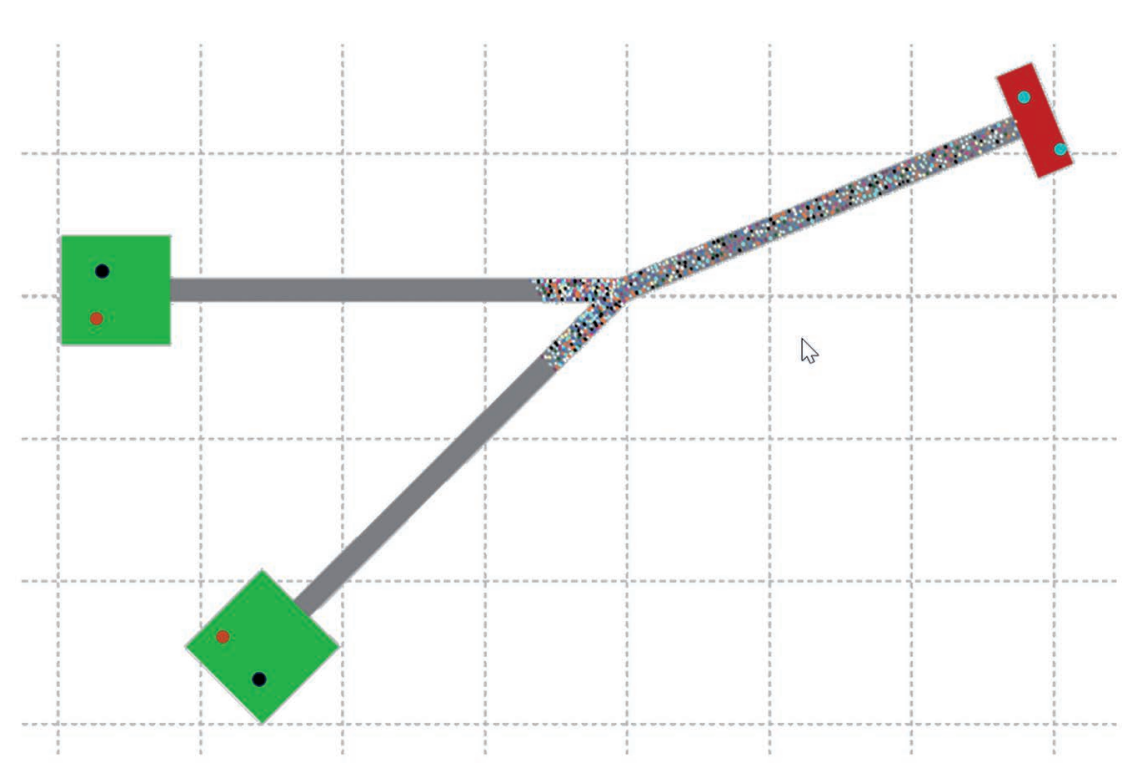

Figure 6. Screen grab of a symmetric scenario at $76 \mathrm{~s}$ from the start of the simulation.

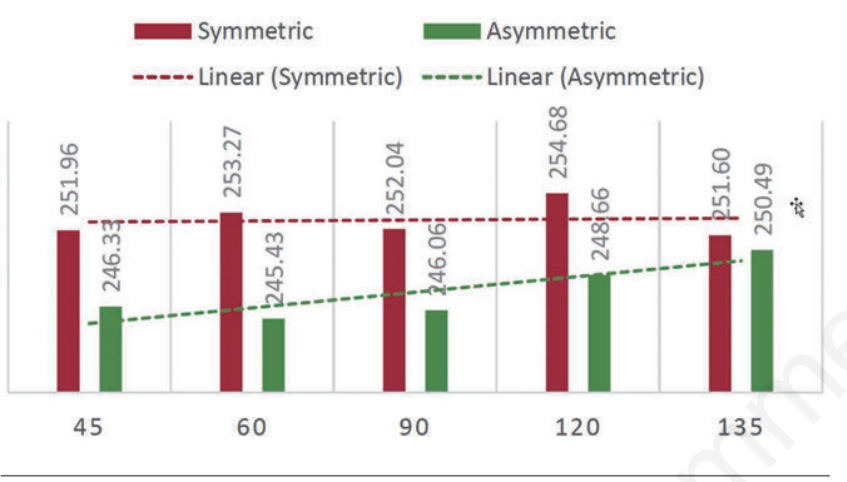

Figure 7. Total evacuation time for desired speeds $=1.32 \mathrm{~m} / \mathrm{s}$.

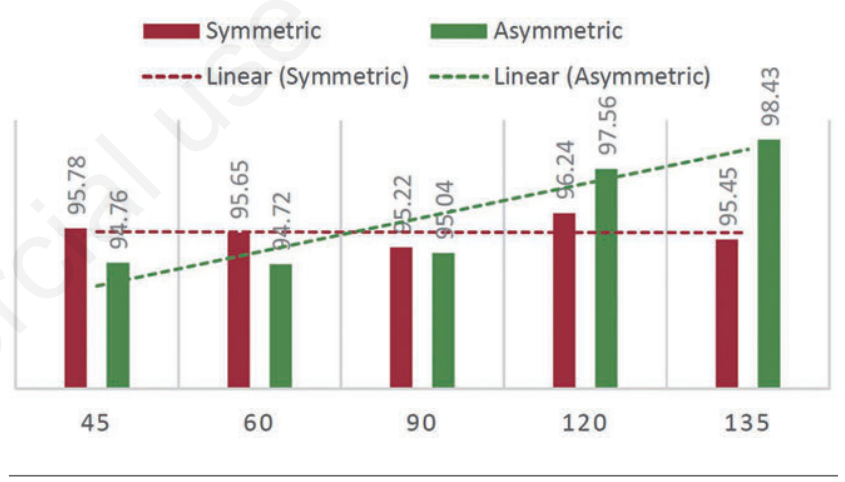

Figure 8. Total evacuation time for desired speeds $=2.50 \mathrm{~m} / \mathrm{s}$.

\section{References}

1. Illera C, Fink M, Hinneberg H, et al. No panic. Escape and panic in buildings architectural basic research in the context of security and safety research, In: Klingsch W, Rogsch C, Schadschneider A, Schreckenberg M, eds. Pedestrian and evacuation dynamics. Berlin: Springer; 2010. pp 733-742.

2. Shiwakoti N, Sarvi M. Enhancing the panic escape of crowd through architectural design. Transport Res Part C: Emerg Technol 2013;37:260-7.

3. Burd M, Shiwakoti N, Sarvi M, Rose G. Nest architecture and traffic flow: large potential effects from small structural features. Ecol Entomol 2010;35:464-8.

4. Duives D, Daamen W, Hoogendoorn S. State-of-the-art crowd motion simulation models. Transport Res Part C 2013;37:193-209.
5. Helbing D, Molnár P. Self-organization phenomena in pedestrian crowds. In: Schweitzer F, ed. Self-organization of complex structures: from individual to collective dynamics. London: Gordon and Breach; 1997. pp 569-577.

6. Hoogendoorn S, Bovy P. Pedestrian route-choice and activity scheduling theory and models. Transport Res Part B: Methodol 2004;38:169-90.

7. Helbing D, Farkas I, Vicsek T. Simulating dynamical features of escape panic. Nature 2000;407:487-90.

8. Helbing D, Molnár P, Farkas I, Bolay K. Self-organizing pedestrian movement. Environ Plann B 2001;28:361-83.

9. Helbing D, Buzna L, Johansson A, Werner T. Self-organized pedestrian crowd dynamics: experiments, simulations, and design solutions. Transport Sci 2005;39:1-24.

10. Shahhoseini Z, Sarvi M. Collective movements of pedestrians: How we can learn from simple experiments with non-human (ant) crowds. PLoS One 2017;12:1-20.

11. Helbing D, Farkas I, Vicsek T. Crowd disasters and simulation of panic situations. In: Bunde A, Kropp J, Schellnhuber HJ, eds. The science of disasters. Berlin, Heidelberg: Springer; 2002. pp 330-350.

12. Dias C, Sarvi M, Shiwakoti N, et al. Investigating collective escape behaviours in complex situations. Safety Sci 2013;60:87-94.

13. Helbing D, Farkas I, Molnár P, Vicsek T. Simulation of pedestrian crowds in normal and evacuation situations. In: Schreckenberg M, Sharma S, eds. Pedestrian and evacuation dynamics. Berlin: Springer; 2002. pp 21-58.

14. Traffex \& Parkex. The 26th international traffic engineering, intelligent trans- 
port systems (ITS), road safety, parking and highway maintenance exhibition. Traffex \& Parkex, 2013 Apr 16-18, NEC, Birmingham. Traffic Eng Control 2013:75-81.

15. Escobar R, De La Rosa A. Architectural design for the survival optimization of panicking fleeing victims. In: Banzhaf W, Ziegler J, Christaller T, et al., eds. Advances in artificial life. Proceedings of the 7th European Conference, ECAL. Lect Notes Comput Sci 2801. Berlin, Heidelberg: Springer; 2003. pp 97-106.
16. Yu W, Chen R, Dong L, Dai S. Centrifugal force model for pedestrian dynamics. Phys Rev E 2005;72:1-7.

17. Frank G, Dorso C. Room evacuation in the presence of an obstacle, Physica A 2011;390:2135-45.

18. Shiwakoti N, Sarvi M, Burd M. Using non-human biological entities to understand pedestrian crowd behaviour under emergency conditions. Safety Sci 2014;66:1-8.

19. Jiang L, Li J, Shen C, et al. Obstacle optimization for panic flow - reducing the tangential momentum increases the escape speed. PLoS One 2014;9:1-15.

20. Johansson A, Helbing D, Shukla PK. Specification of the social force pedestrian model by evolutionary adjustment to video tracking data. Adv Complex Syst 2007;10:271-88.

21. Lima F, Oliveira D, Samed M. Simulação e cenários como preparação para desastres. In: Leiras A, Yoshizaki H, Samed M, Gonçalves M, orgs. Logística humanitária. Rio de Janeiro: Elsevier; 2017. pp 251-272. 